\title{
Left-ventricular mechanical dyssynchrony in the prognosis of dilated cardiomyopathy: Which parameter is more useful?
}

\author{
Weihua Zhou, PhD, ${ }^{\mathrm{a}}$ and Guang-Uei Hung, $M \mathrm{D}^{\mathrm{b}}$ \\ a School of Computing, University of Southern Mississippi, Long Beach, MS \\ b Department of Nuclear Medicine, Chang Bing Show Chwan Memorial Hospital, Changhua, \\ Taiwan
}

Received Mar 20, 2017; accepted Mar 20, 2017

doi: 10.1007/s12350-017-0863-6

\section{See related article, pp. 1677-1687}

Since the invention of the phase analysis technique from ECG-gated myocardial perfusion SPECT by Chen et al in $2005,{ }^{1}$ it has been widely used to measure leftventricular mechanical dyssynchrony (LVMD). Briefly, the phase analysis technique applies a one-dimensional First-harmonic Fourier approximation to the count variation over cardiac frames for each myocardial region on nuclear images and thus generates a 3D phase distribution that describes the timing of LV onset of mechanical contraction over the entire R-R cycle. ${ }^{1,2}$ The technique is fully automated, and has effective temporal resolution of $\sim 15 \mathrm{~ms}$ for a heart rate of $60 /$ minute, high inter- and intra-observer reproducibility, good robustness with camera types, tracer dose, heart rate, and perfusion defects. ${ }^{2,3}$

The commonly used clinical parameters from phase analysis include peak phase (PP), phase standard deviation (PSD), phase bandwidth (PBW), phase histogram skewness, and phase histogram kurtosis. The normal databases consisted of PP $\left(134.5^{\circ} \pm 14.3^{\circ}\right.$ for men and $140.2^{\circ} \pm 14.9^{\circ}$ for women $)$, PSD $\left(14.2^{\circ} \pm 5.1^{\circ}\right.$ for men and $11.8^{\circ} \pm 5.2^{\circ}$ for women $)$, PBW $\left(38.7^{\circ} \pm 11.8^{\circ}\right.$ for men and $30.6^{\circ} \pm 9.6^{\circ}$ for women), skewness $(4.19 \pm 0.68$ for men and $4.60 \pm 0.72$ for women $)$, and

Reprint requests: Weihua Zhou, $\mathrm{PhD}$, School of Computing, University of Southern Mississippi, Long Beach, MS, 39560; weihua.zhou@ usm.edu and Guang-Uei Hung, MD, Department of Nuclear Medicine, Chang Bing Show Chwan Memorial Hospital, Changhua,

Taiwan; 106143@gmail.com

J Nucl Cardiol 2018;25:1688-91.

1071-3581/\$34.00

Copyright (C) 2017 American Society of Nuclear Cardiology. kurtosis (19.72 \pm 7.68 for men and $23.21 \pm 8.16$ for women). ${ }^{1}$ The LVMD parameters have been shown to correlate well with those by tissue Doppler imaging (TDI) echocardiography. ${ }^{4,5}$ Henneman et $\mathrm{al}^{4}$ found that PSD and PWB by phase analysis from SPECT correlated well with LVMD assessed with 2D TDI ( $r=0.80$, $P<0.0001$ and $r=0.89, P<0.0001$, respectively); histogram skewness and kurtosis correlated less well with LVMD on 2D TDI $(r=-0.52, P<0.0001$ and $r=-0.45, P<0.0001$, respectively). Marsan et $\mathrm{al}^{5}$ found that PSD and PBW showed good correlation with $3 \mathrm{D}$ TDI $(r=0.74, \quad P<0.0001$ and $r=0.77$, $P<0.0001$, respectively); histogram skewness showed a poor correlation with 3D TDI $(r=-0.30, P=0.06)$; there was no correlation between histogram kurtosis and 3D TDI ( $r=-0.14, P=0.38)$. PSD and PBW showed the best correlation with the dyssynchrony assessed with echo; hence, they are more commonly used to report the LVMD from gated myocardial perfusion SPECT.

Furthermore, PSD and PBW have been extensively used for the patient selection and prognosis of cardiovascular diseases. In a study with 42 cardiac resynchronization therapy (CRT) patients, ${ }^{6}$ it was demonstrated that responders $(71 \%)$ and non-responders (29\%) had comparable baseline characteristics, except for PSD $\left(56.3^{\circ} \pm 19.9^{\circ}\right.$ vs. $\left.37.1^{\circ} \pm 14.4^{\circ}, P<0.01\right)$ and PBW $\left(175^{\circ} \pm 63^{\circ}\right.$ vs. $\left.117^{\circ} \pm 51^{\circ}, P<0.01\right)$, which were significantly larger in responders compared with non-responders. Moreover, the receiver operating characteristic (ROC) curve analysis demonstrated an optimal cutoff value of $43^{\circ}$ for PSD (sensitivity and specificity of $74 \%$ ) and of $135^{\circ}$ for PBW (sensitivity and specificity of $70 \%$ ) for the prediction of CRT response. In a study with 22 patients who had irreversible ischemic cardiomyopathy and received CRT for at least 12 months, ${ }^{7}$ it was found that PBW was an independent predictor of ventricular arrhythmia. The optimal cutoff value of PBW 
from ROC curve analysis was $>139^{\circ}$. The sensitivity, specificity, positive predictive value, and negative predictive value were $78 \%, 92 \%, 88 \%$, and $86 \%$, respectively. Accordingly, further implantation of defibrillator may be considered for those patients with $\mathrm{PBW}>139^{\circ}$. LVMD was also used to predict major adverse cardiac events (MACE). ${ }^{8}$ It was found that PSD and $\mathrm{PBW}$ were associated with a significantly higher incidence of MACE $(P<0.001)$ and proved to a strong predictor of MACE independent of other known predictors such as perfusion defects or decreased leftventricular ejection fraction (LVEF). Goldberg et $\mathrm{al}^{9}$ enrolled 324 consecutive patients with non-ischemic cardiomyopathy (NICM), LVEF 35\%-50\%, and QRS $<150 \mathrm{~ms}$ and found that the patients who died had a trend toward a higher mean PSD $(P=0.1)$ and PBW $(P=0.08)$. After adjusting for age, hypertension, diabetes, aspirin, beta-blockers, diuretics, QRS, and LVEF, PSD and PBW were independent predictors of all-cause mortality and added incremental prognostic value $\left(P=0.025\right.$ for PSD). In a study by Dr. Wang et al, ${ }^{10}$ severe LVMD was defined as both $\operatorname{PSD}>43^{\circ}$ and $\mathrm{PBW}>135^{\circ}$. In the 48 medically treated dilated cardiomyopathy (DCM) patients, including 14 patients with QRS $\geq 120 \mathrm{~ms}, 12$ cardiac deaths occurred. Compared to survivors, patients with cardiac death had higher PSD $\left(44.0^{\circ} \pm 11.0^{\circ}\right.$ vs. $\left.29.0^{\circ} \pm 15.9^{\circ}, P=0.004\right)$ and $\mathrm{PBW}$ $\left(157.0^{\circ} \pm 37.9^{\circ}\right.$ vs. $\left.105.4^{\circ} \pm 58^{\circ}, P=0.006\right)$.

Nevertheless, it has been reported that PSD and PBW may be influenced by the outliers of phase measurement. ${ }^{11-13}$ PSD may be inappropriate to characterize the widely distributed and sometimes multi-peak distributions in phase histograms; PBW includes nearly a whole range $(95 \%)$ of histogram distribution by excluding possible phase outliers; the phase entropy, which is defined as a term of information theory, reflecting the disorder or uncertainty of a system, is considered to be a promising parameter to characterize the distribution of phase angles. ${ }^{13}$ Phase entropy was reported to be not influenced by the presence of outliers, and it is potentially more sensitive for assessing the index of dispersion than the PSD. ${ }^{11,12}$ In a study for CRT patient selection, ${ }^{14}$ it was demonstrated that a cutoff value of $21^{\circ}$ for PSD with a sensitivity of $90 \%$ and specificity of $74 \%$, a cutoff value of $112^{\circ}$ for PBW with a sensitivity of $72 \%$ and specificity of $70 \%$, and a cutoff of $52 \%$ for entropy with a sensitivity of $90 \%$ and a specificity of $80 \%$ were considered to predict the CRT response.

Noteworthy, in the recent study by Nakajima et al ${ }^{13}$ LVMD parameters, including PSD, PBW, and entropy, measured by different software packages, were compared using normal subjects from the Japanese Society of Nuclear Medicine working group and it was reported that LVMD parameters cannot be interchangeably used among these software packages. The measurement of LVMD may be influenced by several factors, such as, gender, total accumulated count or noise, amount of injected radionuclide, stress or rest, and number of frames per cardiac cycle, myocardial wall sampling algorithms, and configurations.

In the current issue of the journal, Kano et $\mathrm{al}^{15}$ studied the prognostic value of phase entropy by phase analysis of myocardial perfusion SPECT in DCM patients with narrow QRS complex. Two major findings should be noticed:

(1) Phase entropy measured by phase analysis from ECG-gated myocardial perfusion SPECT is a useful parameter in assessing LVMD in patients with DCM. They examined the data accuracy of the selection of LV base site among PSD, PBW, and phase entropy, and confirmed that phase entropy was less influenced by the selection of LV base site than PSD and PBW. Although LV base selection is a major problem influencing the accuracy of phase analysis especially in patients with DCM, phase entropy may have superior accuracy than PSD and PBW. Thus, phase entropy may be more useful in assessing the LVMD in patients with DCM.

(2) Phase entropy is a novel prognostic predictor of adverse cardiac events in patients with mild to moderate DCM and narrow QRS complex (QRS $<120 \mathrm{~ms}$ ). The prolonged QRS duration is associated with a poor prognosis in patients with congestive HF. ${ }^{16}$ LVMD was observed in $27 \%$ to $56 \%$ of patients with a normal QRS complex, ${ }^{17}$ and the QRS duration was reported not to correlate with LVMD. ${ }^{18}$ Therefore, it is important to investigate the prognostic value of LVMD in DCM patient with narrow QRS. The univariate and multivariate Cox regression analysis in this study revealed that the high-phase entropy was a significant and independent predictor of cardiac events. Thus, LVMD by phase entropy has promise for clinicians to more accurately risk-stratify asymptomatic or mildly symptomatic patients with DCM than does the QRS on an ECG. Although the authors strongly emphasized that the phase entropy was a more useful parameter than PSD and PBW in assessing the LVMD in patients with DCM, the superior prognostic value of entropy over PSD and PBW still needs further investigation. In this study, the $P$ values of PSD and PBW were as small as entropy (all $P<0.0001$ ) between the groups of low- and high-phase entropy (Table 2 in $^{15}$ ). Besides, some other software programs for phase analysis of myocardial perfusion SPECT cannot provide the 
measurement of phase entropy and it might be interesting whether such software programs would be limited in assessing the prognosis of DCM patients with narrow QRS. Unfortunately, the authors did not further compare the entropy with PSD and PBW.

However, to further improve the clinical value of LVMD in DCM patients, several significant issues still need to be resolved:

(1) Improvement of the LVMD measurement. Our experience suggests that the major operation which influences LVMD measured by phase analysis from gated myocardial perfusion SPECT is the specification of LV parameters, including LV center, radius, apex, and base. The biggest variation is the selection of base on gated myocardial perfusion SPECT images. The factors that cause ambiguities during the selection of base slices may include the following: (1) the limited resolution of myocardial perfusion SPECT images and the partial volume effect, making the boundary between the LV myocardial base and background unclear; (2) the gating error, patient, and heart motion, and perfusion defect near bases. The severe shape deformation and abnormalities of myocardial perfusion and wall motion in DCM patients even bring more challenges to the parameter specification. It is important to enhance the specification of LV parameters and improve the accuracy of LVMD parameters.

(2) Measurement of LV diastolic dyssynchrony. The phase analysis technique using multi-harmonic Fourier approximation to measure LV diastolic dyssynchrony has been developed and validated by previous studies. ${ }^{19-21}$ A comparative study demonstrated that there was a good correlation between diastolic dyssynchrony by phase analysis from gated myocardial perfusion SPECT and that by TDI ( $r=0.81, P<0.01$ for diastolic PSD; $r=0.75$, $P<0.01$ for diastolic PBW). ${ }^{20}$ In the study by Chen et al, ${ }^{19}$ it was found that the diastolic dyssynchrony parameters were significantly different from the systolic dyssynchrony parameters: the correlation coefficients between the systolic and diastolic dyssynchrony parameters from myocardial perfusion SPECT in 30 normal subjects were 0.53 and 0.61 for PSD and PBW, respectively; the correlation coefficients between the systolic and diastolic dyssynchrony parameters in 121 patients with endstage renal disease and normal LVEF were 0.78 and 0.79 for PSD and PBW, respectively. The study by Hsu et $\mathrm{al}^{21}$ demonstrated that the patients with severe LV systolic dysfunction had severe LV systolic dyssynchrony, but the patients with LV diastolic dysfunction did not necessarily have LV diastolic dyssynchrony. The systolic and diastolic dyssynchrony were physiologically related, but measured different LV mechanisms. As a consequence, diastolic dyssynchrony parameters may be valuable in patients with DCM.

(3) Further clarification of the relationship between LVMD and SERCA2a mRNA levels. The correlation between SERCA2a level and LVMD represented by phase entropy was the strongest among the investigated cardiac parameters, and phase entropy was more closely associated with a decrease in SERCA2a mRNA level rather than the other parameters regarding overall LV dysfunction. However, the pathophysiological mechanisms and cause-effect relationship remain unclear. It should be noted that the mRNA levels may vary with regions and be affected by the selection of biopsy sites. As a result, the SERCA2a mRNA level should be a regional parameter rather than a global index when compared to phase entropy. A regionbased comparison may contribute to the clarification. On the other hand, diastolic dyssynchrony may also be assessed to reveal the pathophysiological mechanism.

\section{References}

1. Chen J, Garcia EV, Folks RD, Cooke CD, Faber TL, Tauxe EL, et al. Onset of left ventricular mechanical contraction as determined by phase analysis of ECG-gated myocardial perfusion SPECT imaging: Development of a diagnostic tool for assessment of cardiac mechanical dyssynchrony. J Nucl Cardiol 2005;12:68795.

2. Chen J, Garcia EV, Bax JJ, Iskandrian AE, Borges-Neto S, Soman P. SPECT myocardial perfusion imaging for the assessment of left ventricular mechanical dyssynchrony. J Nucl Cardiol 2011;18:685-94.

3. Zhou W, Garcia EV. Nuclear image-guided approaches for CRT. Curr Cardiol Rep 2016;18:1-11.

4. Henneman MM, Chen J, Ypenburg C, Dibbets P, Bleeker GB, Boersma E, et al. Phase analysis of gated myocardial perfusion SPECT compared to tissue Doppler imaging for the assessment of left ventricular dyssynchrony. J Am Coll Cardiol 2007;49:1708-14.

5. Marsan NA, Henneman MM, Chen J, Ypenburg C, Dibbets P, Ghio S, et al. Left ventricular dyssynchrony assessed by two 3dimensional imaging modalities: Phase analysis of gated myocardial perfusion SPECT and tri-plane tissue Doppler imaging. Eur J Nucl Med Mol Imaging 2008;35:166-73.

6. Henneman MM, Chen J, Dibbets-Schneider P, Stokkel MP, Bleeker GB, Ypenburg C, et al. Can dyssynchrony as assessed with phase analysis on gated myocardial perfusion SPECT predict response to CRT? J Nucl Med 2007;48:1104-11.

7. Tsai SC, Chang YC, Chiang KF, Lin WY, Huang JL, Hung GU, et al. LV dyssynchrony is helpful in predicting ventricular 
arrhythmia in ischemic cardiomyopathy after cardiac resynchronization therapy: A preliminary study. Medicine 2016;95:e2840.

8. Pazhenkottil AP, Buechel RR, Husmann L, Nkoulou RN, Wolfrum M, Ghadri JR, et al. Long-term prognostic value of left ventricular dyssynchrony assessment by phase analysis from myocardial perfusion imaging. Heart 2011;97:33-7.

9. Goldberg AS, Alraies MC, Cerqueira MD, Jaber WA, Aljaroudi WA. Prognostic value of left ventricular mechanical dyssynchrony by phase analysis in patients with non-ischemic cardiomyopathy with ejection fraction $35 \%-50 \%$ and QRS $<150$ ms. J Nucl Cardiol 2014;21:57-66.

10. Wang L, Yang MF, Cai M, Zhao SH, He ZX, Wang YT. Prognostic significance of left ventricular dyssynchrony by phase analysis of gated SPECT in medically treated patients with dilated cardiomyopathy. Clin Nucl Med 2013;38:510-5.

11. O'Connell JW, Schreck C, Moles M, Badwar N, DeMarco T, Olgin $\mathrm{J}$, et al. A unique method by which to quantitate synchrony with equilibrium radionuclide angiography. J Nucl Cardiol 2005; 12:441-50

12. Van Kriekinge SD, Nishina H, Ohba M, Berman DS, Germano G. Automatic global and regional phase analysis from gated myocardial perfusion SPECT imaging: Application to the characterization of ventricular contraction in patients with left bundle branch block. J Nucl Med 2008;49:1790-7.

13. Nakajima K, Okuda K, Matsuo S, Kiso K, Kinuya S, Garcia EV. Comparison of phase dyssynchrony analysis using gated myocardial perfusion imaging with four software programs: Based on the Japanese Society of Nuclear Medicine working group normal database. J Nucl Cardiol 2016. doi:10.1007/s12350-015-0333-y.

14. Azizian N, Rastgou F, Ghaedian T, Golabchi A, Bahadorian B, Khanlarzadeh $\mathrm{V}$, et al. LV dyssynchrony assessed with phase analysis on gated myocardial perfusion SPECT can predict response to CRT in patients with end-stage heart failure. Res Cardiovasc Med 2014;3:e20720.

15. Kano N, Okumura T, Isobe S, Sawamura A, Watanabe N, Fukaya $\mathrm{K}$, Mori H, et al. Left ventricular phase entropy: Novel prognostic predictor in patients with dilated cardiomyopathy and narrow QRS. J Nucl Cardiol 2017. doi:10.1007/s12350-017-0807-1.

16. Sandhu R, Bahler RC. Prevalence of QRS prolongation in a community hospital cohort of patients with heart failure and its relation to left ventricular systolic dysfunction. Am J Cardiol 2004;93:244-6.

17. Hawkins NM, Petrie MC, MacDonald MR, Hogg KJ, McMurray JJ. Selecting patients for cardiac resynchronization therapy: Electrical or mechanical dyssynchrony? Eur Heart J 2006;27:1270-81.

18. Yu CM, Lin H, Zhang Q, Sanderson JE. High prevalence of left ventricular systolic and diastolic asynchrony in patients with congestive heart failure and normal QRS duration. Heart 2003;89:54-60.

19. Chen J, Kalogeropoulos AP, Verdes L, Butler J, Garcia EV. Leftventricular systolic and diastolic dyssynchrony as assessed by multi-harmonic phase analysis of gated SPECT myocardial perfusion imaging in patients with end-stage renal disease and normal LVEF. J Nucl Cardiol 2011;18:299-308.

20. Boogers MJ, Chen J, Veltman CE, van Bommel RJ, Mooyaart EA, Al Younis I, et al. Left ventricular diastolic dyssynchrony assessed with phase analysis of gated myocardial perfusion SPECT: A comparison with tissue Doppler imaging. Eur J Nucl Med Mol Imaging 2011;38:2031-9.

21. Hsu TH, Huang WS, Chen CC, Hung GU, Chen TC, Kao CH, et al. Left ventricular systolic and diastolic dyssynchrony assessed by phase analysis of gated SPECT myocardial perfusion imaging: A comparison with speckle tracking echocardiography. Ann Nucl Med 2013;27:764-71. 